\title{
Qualitative and quantitative assessment of pendelluft: a simple method based on electrical impedance tomography
}

\author{
Ling Sang ${ }^{1 \#}$, Zhanqi Zhao ${ }^{2,3 \#}$, Po-Jen Yun ${ }^{4}$, Inéz Frerichs ${ }^{5}$, Knut Möller ${ }^{3}$, Feng Fu ${ }^{2}$, Xiaoqing Liu ${ }^{1}$, \\ Nanshan Zhong ${ }^{1}$, Yimin Li $^{1}$ \\ ${ }^{1}$ Department of Critical Care Medicine, The First Affiliated Hospital of Guangzhou Medical University, Guangzhou Medical University, State Key \\ Lab of Respiratory Diseases, Guangzhou Institute of Respiratory Health, Guangzhou, China; ${ }^{2}$ Department of Biomedical Engineering, Fourth \\ Military Medical University, Xi'an, China; ${ }^{3}$ Institute of Technical Medicine, Furtwangen University, Villingen-Schwenningen, Germany; ${ }^{4}$ Division \\ of Thoracic Surgery, Department of Surgery, Tri-Service General Hospital, Taipei; ${ }^{5}$ Department of Anesthesiology and Intensive Care Medicine, \\ University Medical Center of Schleswig-Holstein Campus Kiel, Kiel, Germany \\ Contributions: (I) Conception and design: X Liu, N Zhong, Y Li; (II) Administrative support: None; (III) Provision of study materials or patients: L \\ Sang, Z Zhao, PJ Yun; (IV) Collection and assembly of data: L Sang, Z Zhao, PJ Yun; (V) Data analysis and interpretation: I Frerichs, K Möller, F \\ Fu; (VI) Manuscript writing: All authors; (VII) Final approval of manuscript: All authors. \\ \#These authors contributed equally to this work. \\ Correspondence to: Dr. Nanshan Zhong; Dr. Yimin Li. Department of Critical Care Medicine, The First Affiliated Hospital of Guangzhou Medical \\ University, Guangzhou Medical University, State Key Lab of Respiratory Diseases, Guangzhou Institute of Respiratory Health, Guangzhou, China. \\ Email: nanshan@vip.163.com; dryiminli@vip.163.com.
}

Background: Pendelluft, defined as asynchronous alveolar ventilation, is caused by different regional time constants or dynamic pleural pressure variations. The aim of the present study was to propose a simple method to evaluate pendelluft based on electrical impedance tomography (EIT). The efficacy of this method was demonstrated in well-known pendelluft scenarios in 6 patients.

Methods: Two patients with flail chest after accidents, two patients with acute respiratory distress syndrome (ARDS) and two patients with acutely exacerbated obstructive lung disease were prospectively included. EIT measurements were performed before and after surgery (in patients with flail chest, who had video-assisted thoracoscopic surgery with ribs fixation), or at two different levels of positive end-expiratory pressure (PEEP; ARDS patients), or two different time points (obstructive lung disease). Pendelluft was assessed by regional phase shift (defined as time difference between global and regional impedance-time curves) and amplitude differences (defined as the impedance difference between sum of all regional tidal variation and the global tidal variation).

Results: In patients with flail chest, pendelluft diminished several days after surgery (pendelluft amplitude normalized to tidal impedance variation reduced from $88 \%$ to $2 \%$ in one patient, $12 \%$ to $2 \%$ in the other). Increased PEEP reduced the amplitude of pendelluft (from $3 \%$ to $0 \%$ in one patient, $20 \%$ to $2 \%$ in the other) but not necessarily the phase shifts (average time differences were $<0.1$ second for both patients for both ins- and expiration) in ARDS patients. Pendelluft assessment in obstructive lung diseases reflected the change in airway resistance (from $5 \%$ to $1 \%$ in one patient after broncholytic medication administration, as airway resistance fell from 15 to $11 \mathrm{cmH}_{2} \mathrm{O} / \mathrm{L} / \mathrm{s}$; from $9 \%$ to $35 \%$ in the other patient with acute exacerbation, the corresponding airway resistance increased from 15 to $22 \mathrm{cmH}_{2} \mathrm{O} / \mathrm{L} / \mathrm{s}$ ).

Conclusions: The proposed EIT-based method can be used to evaluate the degree of pendelluft in dimension of phase shift and amplitude difference.

Keywords: Electrical impedance tomography (EIT); pendelluft; acute respiratory distress syndrome (ARDS); obstructive lung disease; flail chest

Submitted May 22, 2020. Accepted for publication Aug 12, 2020.

doi: 10.21037/atm-20-4182

View this article at: http://dx.doi.org/10.21037/atm-20-4182 


\section{Introduction}

Pendelluft, defined as asynchronous alveolar ventilation, is caused by different regional time constants or dynamic pleural pressure variations. In patients with obstructive lung diseases (e.g., chronic obstructive lung disease COPD), time constant of the respiratory system varies significantly in different lung regions, which leads to the pendelluft phenomenon (1). In spontaneously breathing patients with injured lung [e.g., acute respiratory distress syndrome (ARDS)], non-uniform transmission of pleural pressure generated by diaphragmatic contraction is the main reason for pendelluft (2). In the extreme cases such as flail chest, pendelluft volume can be as high as $12.5 \%$ of the total volume passing through an airway (3).

Since pendelluft could be potentially harmful by introducing local overstretch, tidal recruitment and inflammation (4), the detection of pendelluft is warranted so that the treatment or ventilation strategies could be adjusted accordingly. In early days with limited technology options, pendelluft was observed in surgeries with an open hemithorax (5). The ventilation asynchrony could only be detected between left to right lungs in experimental settings (6). The degree of pendelluft was also assessed by calculating the correlated lung mechanics (7). Pathological alterations in lungs could be observed by computed tomography and magnetic resonance imaging $(8,9)$, which provide indirect information of regional time constants. In a previous study, positron imaging technique was used to capture the clearance of the tracer nitrogen-13 to calculate the pendelluft (10). In a recent study, a microphone array was used to detect pendelluft (1). In experimental settings, darkfield microscopy and multispectral oximetry were also applied to assess the effect of sighs on pendelluft (11).

The methods described above could not be used at the bedside to identify and continuously measure the pendelluft. Electrical impedance tomography (EIT) is a novel imaging technique that allows real time detection of pendelluft at the bedside (12). More and more studies using EIT to detect pendelluft have been published recently (13-15). However, the EIT methods used in these studies either compared impedance-time curves from different regions of interest (ROIs), which is time-consuming and may miss the pendelluft depending on the division of ROIs (13), or required an interruption of normal ventilation (14), or no quantitative measurement was provided (15).

The aim of the present study was to propose a simple EIT-based method to evaluate pendelluft. The efficacy of the method was demonstrated in well-known pendelluft scenarios in various patients. We present the following article in accordance with the MDAR reporting checklist (available at http://dx.doi.org/10.21037/atm-20-4182).

\section{Methods}

\section{Patients and measurements}

The trial was conducted in accordance with the Declaration of Helsinki (as revised in 2013). The patients were from undergoing studies that were approved by institutional ethics review boards of the first affiliated hospital of Guangzhou Medical University, Guangzhou (2017-04) and of Tri-Service General Hospital, Taipei (2-106-05-105). Informed consent was obtained from all patients or next of kin before data were included into the study.

In total 6 patients were included representing 3 scenarios with well-known pendelluft phenomenon.

Scenario 1: two patients had flail chest after accidents (left side rib fractures). The patients had video-assisted thoracoscopic surgery with ribs fixation, under general anesthesia. EIT measurements were performed in supine position before and 4-5 days after the surgery. Patients were spontaneously breathing without any ventilation support. Visual analog scale pain scoring (VAS) was used to assess the pain level (16).

Scenario 2: two patients with ARDS under mechanical ventilation were included. EIT data were recorded at two different steps of positive end-expiratory pressure (PEEP). The diagnosis criteria for ARDS were applied according to the published guideline (17). The patients were ventilated in the supine position with the tidal volume (VT) set to $6 \mathrm{~mL} / \mathrm{kg}$ predicted body weight (Evita 4, Dräger Medical, Lübeck, Germany). The fraction of inspired oxygen $\left(\mathrm{FiO}_{2}\right)$ was 0.5 .

Scenario 3: two patients with acute exacerbation of obstructive lung disease were examined. They were under mechanical ventilation. In one patient with asthma, EIT measurements were performed before and 60 minutes after administration of long-acting $\beta 2$ adrenergic receptor agonist (LABA) and steroid. In the other patient with COPD, EIT data were recorded on two different days, whereby on the latter day, the patient's airway resistance was significantly increased. The diagnosis criteria for COPD and for asthma were performed according to the published guidelines $(18,19)$, respectively. Patients were ventilated under assistcontrol mode with EVITA 4 (Dräger Medical, Lübeck, 
Germany). Ventilator settings were: tidal volume $8 \mathrm{~mL} / \mathrm{kg}$ predicted body weight, PEEP $5 \mathrm{cmH}_{2} \mathrm{O}$, and inspiratory to expiratory time ratio $1: 2$.

To conduct the EIT measurements, an EIT belt with 16 electrodes was placed around the thorax at the $4-5$ th intercostal spaces level (PulmoVista 500, Dräger Medical, Lübeck, Germany). All patients received standard care and no other research related interventions were performed during the measurements. EIT measurements were continuously recorded at $20 \mathrm{~Hz}$. The EIT data were digitally filtered using a low-pass filter with a cut-off frequency of $0.67 \mathrm{~Hz}$ to eliminate cardiac related impedance changes. Further, the data were analyzed offline using customized software, programmed with MATLAB R2015 (the MathWorks Inc., Natick, MA).

\section{EIT-based pendelluft measures}

The global ventilation map was calculated by subtracting the inspiration-begin from the expiration-begin image based on the global impedance-time curve, which represents the global EIT signal variation during tidal breathing. The global tidal images of one minute were averaged to increase the signal to noise ratio.

$$
V_{G_{-} i}=\frac{1}{N} \sum_{n=1}^{N}\left(\Delta Z_{i, E X P, n}-\Delta Z_{i, I N S, n}\right)
$$

where $V_{G_{-} i}$ is the pixel $i$ in the global ventilation image; $N$ is the number of breaths within the analyzed period; INS and EXP are the time points of global inspiration-begin and global expiration-begin, respectively. $\Delta Z_{i, I N S}$ and $\Delta Z_{i, E X P}$ are the corresponding pixel values in the raw EIT image.

The regional ventilation map was calculated by subtracting the inspiration-begin from the expiration-begin on individual pixel impedance-time curves, which represent the regional variation during tidal breathing. The regional tidal images of one minute were averaged to increase the signal to noise ratio.

$$
V_{R_{-} i}=\frac{1}{N} \sum_{n=1}^{N}\left(\Delta z_{i, \exp , n}-\Delta z_{i, i n s, n}\right)
$$

where $V_{R_{-} i}$ is the pixel $i$ in the regional ventilation image; $N$ is the number of breaths within analyzed period; ins and exp are the time points of inspiration-begin and global expiration-begin on individual pixel impedance-time curves, respectively. $\Delta z_{i, i n s}$ and $\Delta z_{i, e x p}$ are the corresponding pixel values.

Regional time difference at inspiration-begin is defined as

$$
T_{\text {Ins } \_}=\frac{1}{N} \sum_{n=1}^{N}\left(\text { ins }_{i, n}-I N S_{i, n}\right) \quad i \in \text { lung }
$$

Regional time difference at expiration-begin is defined as

$$
T_{E_{\text {Exp }} i}=\frac{1}{N} \sum_{n=1}^{N}\left(\exp _{i, n}-E X P_{i, n}\right) \quad i \in \text { lung }
$$

The regional time difference has a unit of second.

Pendelluft amplitude is defined as in Eq. 5. For each pixel, the impedance difference at the global time points (end-inspiration/expiration) was subtracted by the regional tidal variation of the corresponding pixel.

$A_{i}=\frac{1}{N} \sum_{n=1}^{N}\left(V_{R_{-} i, n}-V_{G_{-} i, n}\right) / T V \times 100 \% \quad i \in$ lung

where $T V$ represents the global tidal variation. If tidal volume of each breath is obtained (e.g., when the ventilator is connected to the EIT device), the amplitude can be normalized to milliliters. The parameters of the EIT measures are illustrated in Figure 1.

\section{Statistical analysis}

The data analysis was conducted using MATLAB 2015a (The MathWorks Inc., Natick, MA, USA). Means of analyzed parameters were presented.

\section{Results}

\section{Scenario 1: patients with flail chest}

For patient No. 1, EIT measurement was performed before and 5 days after the surgery (Figure 2). Out-of-phase ventilation was observed at the affected left chest side with fractured ribs. Pain VAS significantly improved after surgery (from 9 to 1). The pendelluft amplitude reduced from $88 \%$ to $2 \%$. Similar trend of pendelluft improvement (Figure 3) was found in patient No. 2 without an improvement of pain VAS (both 4 before and after surgery). Although visually the $T_{\text {Exp }}$ values in these two patients were dissimilar, the average values were 0.05 second, which was limited due to the temporal resolution of EIT recording $(20 \mathrm{~Hz})$.

\section{Scenario 2: ARDS patients with PEEP changes}

Upon ICU admission, patient No. 3. had an initial $\mathrm{PaO}_{2} /$ $\mathrm{FiO}_{2}$ of $176 \mathrm{mmHg}$, APACHE II 23, SOFA 6. Patient No. 




Figure 1 Generation of EIT-based pendelluft measures. Top: the global impedance-time curve. INS and EXP are the time points of global inspiration-begin and global expiration-begin, respectively. Bottom: impedance-time curves from two pixels with large ventilation shift. ins and $\exp$ are the time points of inspiration-begin and expiration-begin on individual pixel impedance-time curves, respectively. $\mathrm{V}_{\mathrm{G}}$ and $\mathrm{V}_{\mathrm{R}}$ are global and regional tidal variations, respectively. AU, arbitrary unit. S, time difference in second. /TV\%, pendelluft amplitude to tidal variation ratio in percent.
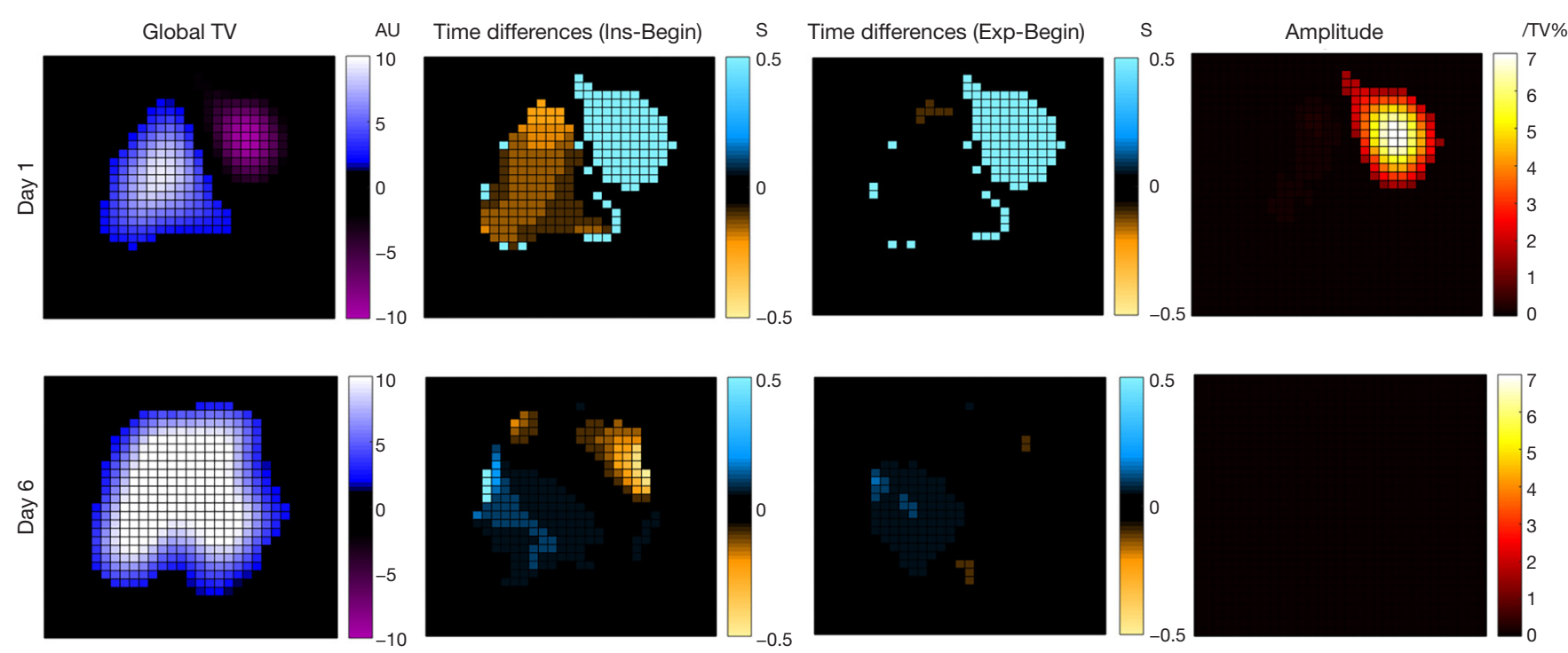

Figure 2 Patient No. 1 with flail chest (female, 63 years old, $160 \mathrm{~cm}$ height and $75 \mathrm{~kg}$ weight, left-sided rib fractures). The patient had video-assisted thoracoscopic surgery with ribs fixation, under general anesthesia. First row, day 1, before surgery. Second row, day 6, 5 days after surgery. First column, global tidal variation. Low and high ventilated regions are marked in dark and light blue, respectively. Outof-phase impedance changes are marked in purple. Second column, time difference functional EIT image for inspiration begin. Third column, time difference functional EIT image for expiration begin. Orange and blue mark the early and delayed ventilation compared to the global curve. Fourth column, amplitude of pendelluft. Red to yellow show the increase of pendelluft amplitude. AU, arbitrary unit. S, time difference in second. /TV\%, pendelluft amplitude to tidal variation ratio in percent. 

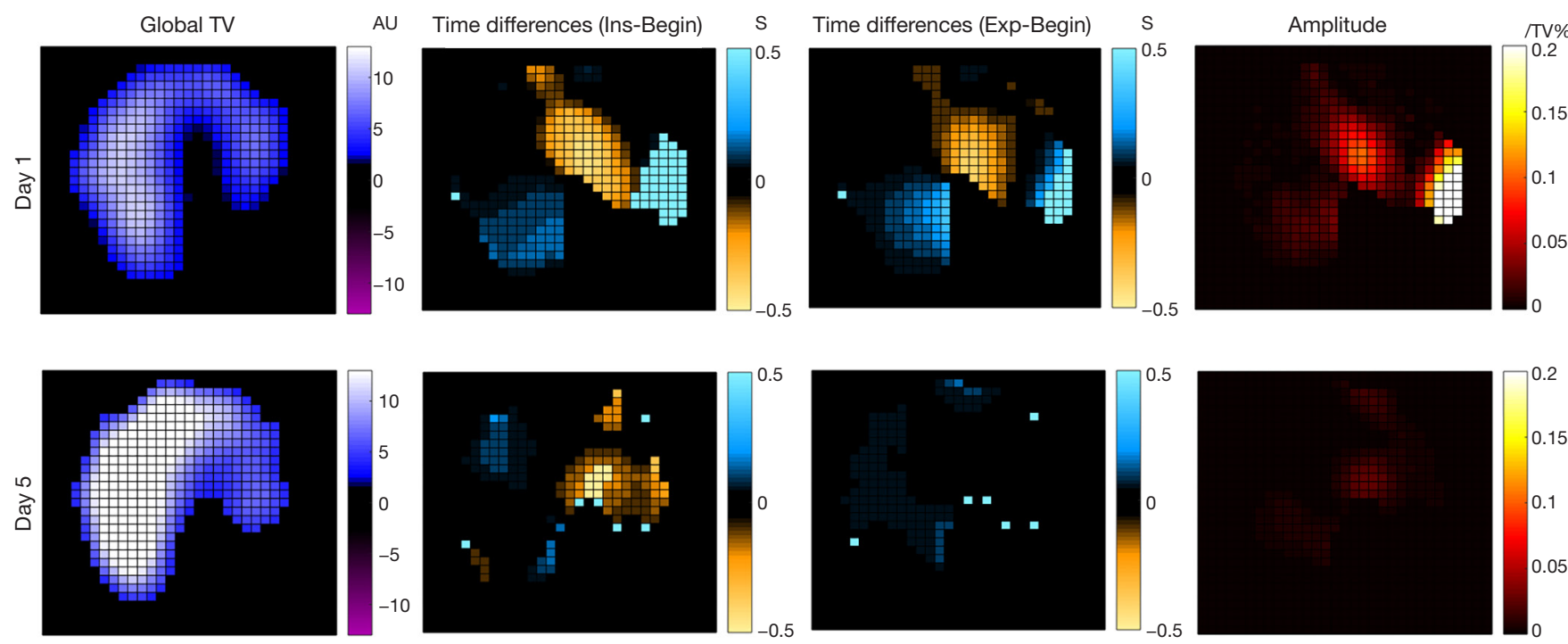

Figure 3 Patient No. 2 with flail chest (male, 49 years old, $170 \mathrm{~cm}$ height and $68 \mathrm{~kg}$ weight, left-sided rib fractures). The patient had videoassisted thoracoscopic surgery with ribs fixation, under general anesthesia. First row, day 1, before surgery. Second row, day 5 , 4 days after surgery. First column, global tidal variation. Low and high ventilated regions are marked in dark and light blue, respectively. Out-of-phase impedance changes are marked in purple. Second column, time difference functional EIT image for inspiration begin. Third column, time difference functional EIT image for expiration begin. Orange and blue mark the early and delayed ventilation compared to the global curve. Fourth column, amplitude of pendelluft. Red to yellow show the increase of pendelluft amplitude. AU, arbitrary unit. S, time difference in second. /TV\%, pendelluft amplitude to tidal variation ratio in percent.

4 had an initial $\mathrm{PaO}_{2} / \mathrm{FiO}_{2}$ of $208 \mathrm{mmHg}$, APACHE II 22, SOFA 12. The efficacy of recruitment by increasing PEEP was significantly different in these two patients. An increase of $10 \mathrm{cmH}_{2} \mathrm{O}$ in PEEP did not increase the tidal ventilation or affect the ventilation distribution pattern in patient No. 3 (Figure 4, first column). On the other hand, gravity-dependent regions were recruited in patient No. 4 (Figure 5, first column). Higher PEEP reduced the pendelluft amplitude (Figures 4,5, last column) but not necessarily the phase shift (Figure 5, second and third columns). For patient No. 4, the real time parameters were recorded simultaneously from the ventilator, so that $\mathrm{TV}$ was normalized to tidal volume (Figure 5, first column, unit in milliliter) and the pendelluft amplitude could be quantitatively calculated.

\section{Scenario 3: patients with obstructive lung diseases}

In patient No. 5 with acute exacerbation of asthma, pendelluft improved with regard to both the phase and the amplitude (Figure 6, Table 1) after administration of medication. Airway resistance reduced from 15 to $11 \mathrm{cmH}_{2} \mathrm{O} / \mathrm{L} / \mathrm{s}$. In patient No. 6 with acute exacerbation of
COPD, pendelluft between the left and right lungs became more significant with the disease development (Figure 7, Table 1), whereas the corresponding airway resistances increased from 15 to $22 \mathrm{cmH}_{2} \mathrm{O} / \mathrm{L} / \mathrm{s}$. Real time parameters from the ventilator were recorded simultaneously for both patients, so that TV could be normalized to tidal volume (Figures 6,7, first column, unit in milliliter) and the pendelluft amplitude quantitatively calculated.

\section{Discussion}

In the present study, we proposed an EIT-based method to assess the pendelluft in subjects breathing spontaneously or with ventilator support. The method captured both the phase shifts (time difference between global and regional impedance-time curves) and the amplitude of pendelluft. When the tidal volume is recorded simultaneously, the amplitude of pendelluft could be calculated quantitatively.

Subject with flail chest was one of the earliest scenarios that confirmed the existence of the pendelluft phenomenon (3). One may suspect that the ventilation asynchrony could be caused by the pain of subject and the subsequent reduction of respiratory strength at the chest 

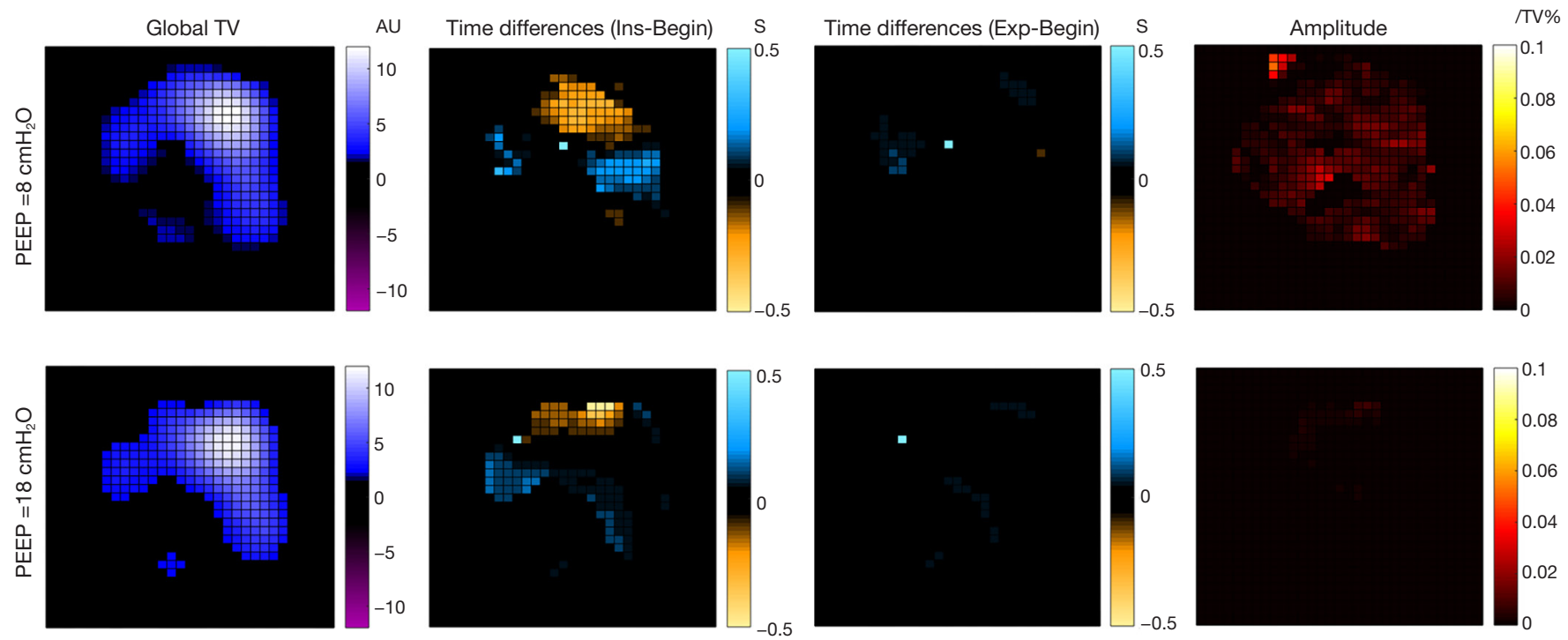

Figure 4 Patient No. 3 with ARDS (male, 70 years old, $165 \mathrm{~cm}$ height and $67 \mathrm{~kg}$ weight). First row, PEEP $8 \mathrm{cmH} \mathrm{O}_{2} \mathrm{O}$ Second row, PEEP $18 \mathrm{cmH}_{2} \mathrm{O}$. First column, global tidal variation. Low and high ventilated regions are marked in dark and light blue, respectively. Out-ofphase impedance changes are marked in purple. Second column, time difference functional EIT image for inspiration begin. Third column, time difference functional EIT image for expiration begin. Orange and blue mark the early and delayed ventilation compared to the global curve. Fourth column, amplitude of pendelluft. Red to yellow show the increase of pendelluft amplitude. AU, arbitrary unit. S, time difference in second. /TV\%, pendelluft amplitude to tidal variation ratio in percent. PEEP, positive end-expiratory pressure.
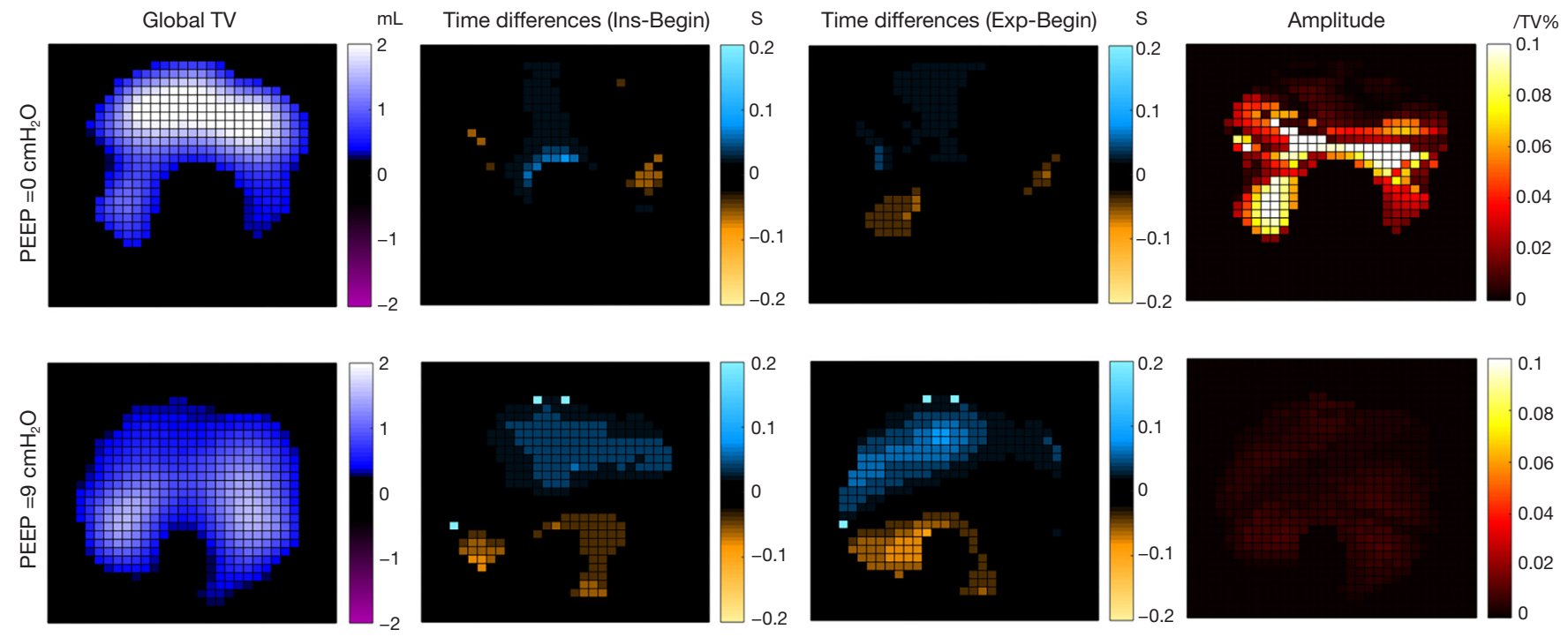

Figure 5 Patient No. 4 with ARDS (male, 76 years old, $175 \mathrm{~cm}$ height and $53 \mathrm{~kg}$ weight). First row, PEEP $0 \mathrm{cmH}_{2} \mathrm{O} . \mathrm{Second}$ row, PEEP $9 \mathrm{cmH}_{2} \mathrm{O}$. First column, global tidal variation. Low and high ventilated regions are marked in dark and light blue, respectively. Out-of-phase impedance changes are marked in purple. Second column, time difference functional EIT image for inspiration begin. Third column, time difference functional EIT image for expiration begin. Orange and blue mark the early and delay ventilation compared to the global curve. Fourth column, amplitude of pendelluft. Red to yellow show the increase of pendelluft amplitude. AU, arbitrary unit. S, time difference in second. /TV\%, pendelluft amplitude to tidal variation ratio in percent. PEEP, positive end-expiratory pressure. 

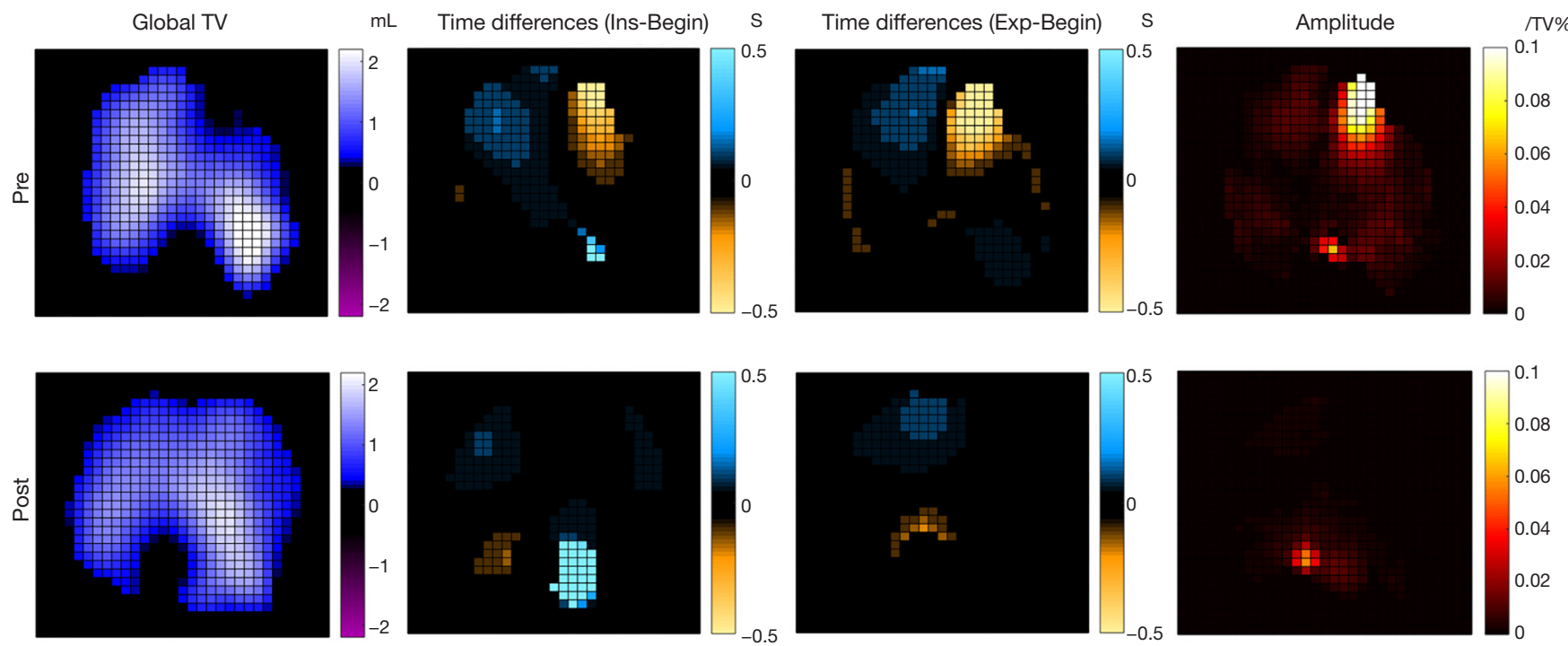

Figure 6 Patient No. 5 with acute exacerbation of asthma (female, 24 years old, $165 \mathrm{~cm}$ height and $60 \mathrm{~kg}$ weight). First row, before steroid and LABA administration. Second row, 60 minutes after the medication. First column, global tidal variation (TV). Low and high ventilated regions are marked in dark and light blue, respectively. Out-of-phase impedance changes are marked in purple. Second column, time difference functional EIT image for inspiration begin. Third column, time difference functional EIT image for expiration begin. Orange and blue mark the early and delayed ventilation compared to the global curve. Fourth column, amplitude of pendelluft. Red to yellow show the increase of pendelluft amplitude. AU, arbitrary unit. S, time difference in second. /TV\%, pendelluft amplitude to tidal variation ratio in percent.

Table 1 Summary of EIT-based measures

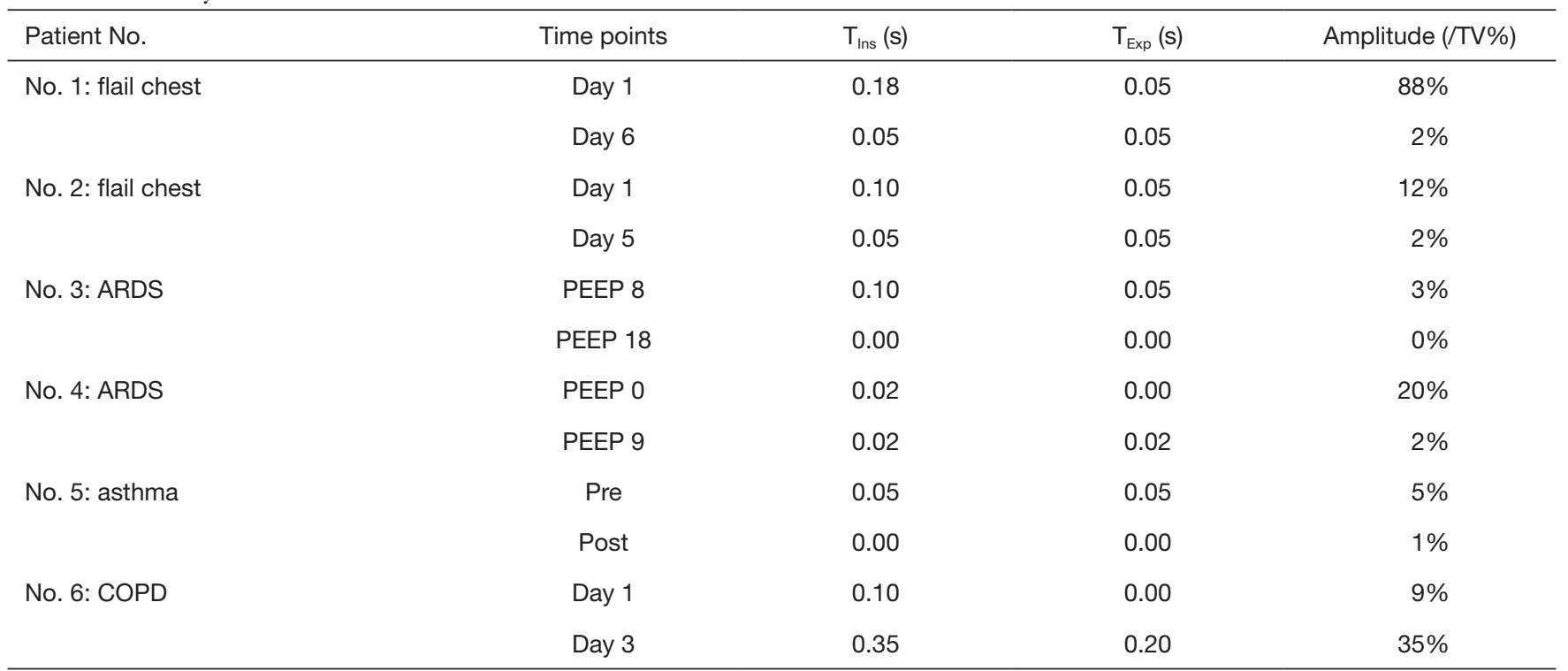

EIT, electrical impedance tomography. ARDS, acute respiratory distress syndrome. COPD, chronic obstructive lung disease. $T_{\text {Ins }}$ and $T_{E x p}$ are the average values of absolute time differences for inspiration and expiration begin, respectively. /TV\%, pendelluft amplitude to tidal variation ratio in percent. PEEP, positive end-expiratory pressure. 

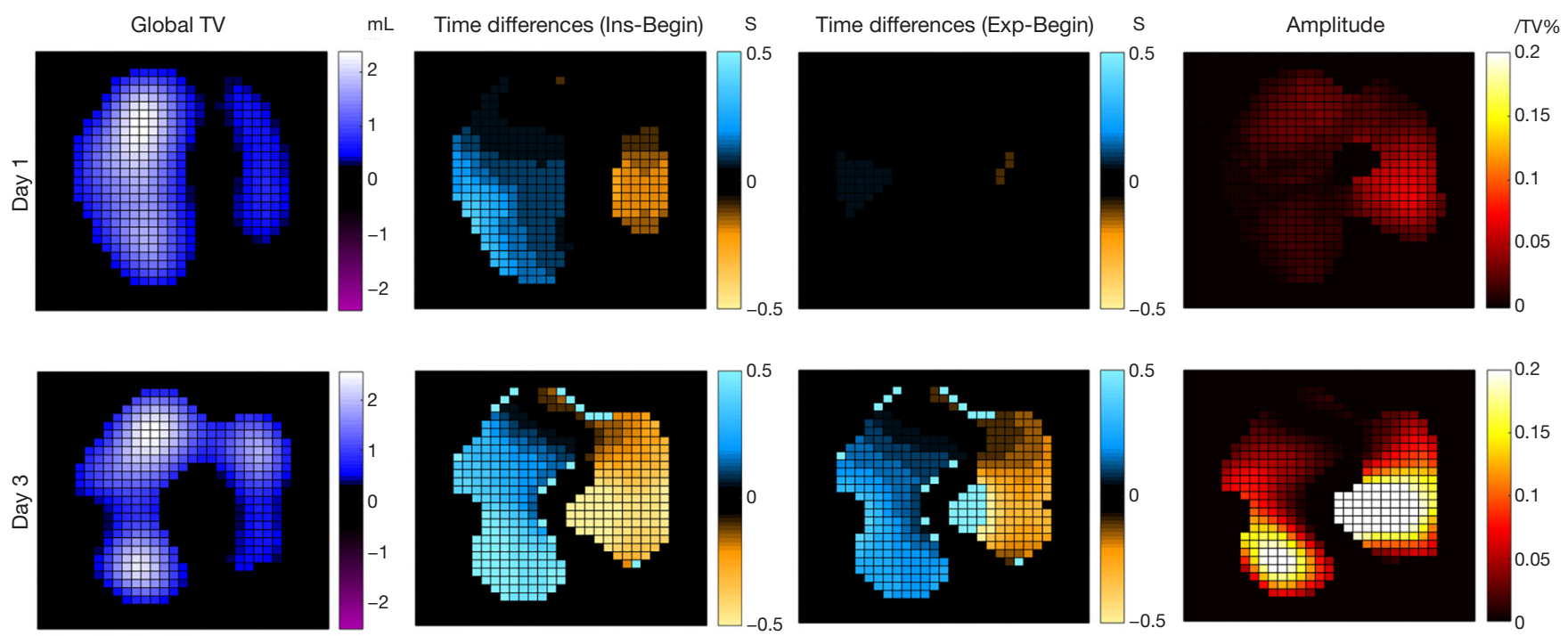

Figure 7 Patient No. 6 with acute exacerbation of chronic obstructive lung disease (female, 91 years old, $141 \mathrm{~cm}$ height and $41 \mathrm{~kg}$ weight). First row, day 1 in ICU. Second row, day 3 in ICU. First column, global tidal variation. Low and high ventilated regions are marked in dark and light blue, respectively. Out-of-phase impedance changes are marked in purple. Second column, time difference functional EIT image for inspiration begin. Third column, time difference functional EIT image for expiration begin. Orange and blue mark the early and delayed ventilation compared to the global curve. Fourth column, amplitude of pendelluft. Red to yellow show the increase of pendelluft amplitude. $\mathrm{AU}$, arbitrary unit. S, time difference in second. /TV\%, pendelluft amplitude to tidal variation ratio in percent.

side with rib fractures. According to the present finding, the reduction of pendelluft might not be associated with pain directly (Table 1). Besides, we were worried about the tolerance of EIT measurements in this group of subjects due to chest pain. A recent study indicated that EIT electrode belt may have influence on the lung function in sitting position (20). That study did not test the influence of the electrode belt in supine position, where the force of the belt posed on the chest could be reduced to get satisfactory EIT results. Indeed, the two currently examined subjects with flail chest tolerated the EIT measurements well. The average values of $T_{\operatorname{Exp}}$ were not very sensitive due to the sampling rate of EIT measurements. Higher sampling rate of $50 \mathrm{~Hz}$ would increase the temporal resolution to 0.02 second. To quantitatively assess pendelluft in spontaneously breathing patients, simultaneous spirometry measurement could be conducted, which would provide tidal volume for reference.

It was found that spontaneous breathing in ARDS increased transpulmonary pressure, which may worsen lung injury (21). Recent research focused on pendelluft in lung injury patients and how to minimize the adverse effect of spontaneous breathing $(13,15)$. Although EIT may help to identify pendelluft at the bedside, physicians needed to define a ROI that contained pendelluft (13). A mixture of regions with pendelluft and normally ventilated alveoli within one ROI would reduce the detection of pendelluft in the impedance-time curves. On the contrary, our method quantifies "pendelluft" based on single pixel analysis, which enables high spatial resolution. Besides, only the phase shifts (time difference between global and regional impedancetime curves) were evaluated. Santini et al. proposed a method to assess the degree of pendelluft by calculating the regional impedance changes during an inspiratory hold between the beginning and the end of the pause (14). This method required control ventilation and interruption of the normal breathing pattern. Only the pendelluft amplitude at the end of inspiration could be captured by this method. The method proposed in the present study, on the other hand, evaluated the phase shifts and amplitude at the same time, giving a more complex understanding of pendelluft associated with PEEP changes. The effects of PEEP changes on pendelluft may vary in regard to phase shifts and amplitude (Table 1). They may also depend on the response to recruitment (Figures 4,5), which needs further investigation with a larger group of study participants. 
The time difference in inspiration and expiration in ARDS patients could be caused by various opening and closing pressures between different lung regions (22). We acknowledge that the pendelluft calculated with present method does not distinguish the causes, whether by various recruitment/derecruitment pressures or by various airway resistances.

Airway resistance significantly increases in obstructive lung diseases, which leads to a high time constant that causes the pendelluft phenomenon (1). An EIT-based method for regional expiratory time constants was proposed, which suggested that breath-by-breath calculation of regional time constants was feasible (23). We also found that the degree of pendelluft changed along with the airway resistance (Table 1). The treatment effect with steroid and LABA was clearly showed in the regional time difference and amplitude maps (Figure 6). Exacerbation could be visualized and quantitatively assessed by our proposed method (Figure 7). The exact correlation between the pendelluft assessment and the change of airway resistance and time constant needs to be investigated in further studies. Recent EIT applications on obstructive lung diseases suggested that EIT has the potential to evaluate the treatment efficacy both qualitatively and quantitatively (24-26). Pendelluft assessment proposed in the present study enlarges the EIT application fields in such patient group.

The definition of pendelluft is not unified, whether flow coming from outside (ventilator or room air) should be absent or not $(2,14)$. In our opinion, a redistribution of external flow should be also considered reflecting the degree of pendelluft, the proposed method does not require zeroing of external flow. As a limitation of the present study, the results of the proposed method were not validated against any references. Unfortunately, no well-established bedside techniques are capable to assess pendelluft with sufficient spatial and temporal resolution as EIT. We may design scenarios to calculate pendelluft using previously suggested EIT-based methods; however, no objective measures could be used to judge the superiority of the methods. To design the study and to examine the sensitivity of the proposed method, we recruited 6 patients in 3 scenarios, which covered major occurrences of the pendelluft phenomenon. In each scenario, we measured patients at 2 different time points, where the changes of pendelluft in these events were previously documented. Different EIT-based methods were discussed and compared indirectly based on their application prerequisites. As the signal of interest ("pendelluft") is small, the present method averages the signal of one minute for calculation. By using this invention, even very small signals that would be missed by analyzing only single breaths can be detected.

As a proof of concept study, the number of patients recruited was low and not all scenarios with pendelluft phenomena were included [e.g., weaning patients (27)]. Nevertheless, we were able to demonstrate the application of the pendelluft assessment with these representative cases. The possibility to analyze time dependent changes within the lung allows a glimpse on a phenomenon, which has been a long-lasting challenge to lung physiology. Visualization of inhomogeneity which leads to what has been called "pendelluft" may give completely new insights to the quick dynamics within the ventilated lung. $U p$ to now, EIT-related research mainly focuses on the ability of bedside monitoring or the advantage of radiation-free imaging. Our method emphasizes the dynamic phenomena within the ventilating lung, which may open the view to new information that has not been available without EIT.

\section{Conclusions}

The proposed EIT-based method can be used to evaluate the degree of pendelluft in the dimension of phase shift and amplitude difference. EIT has the potential to guide ventilator settings and treatments at the bedside.

\section{Acknowledgments}

Funding: This work was partially supported by the Natural Science Foundation of Guangdong Province (2020A151501964); National Science and Technology Major Project (No. 2017ZX10204401); Self-determined Project of GIRH (2019GIRHQ05); the Everest Program of AFMU (Grant No. 2019ZFB002); BMBF MOVE (FKZ 13FH628IX6) and H2020 MCSA Rise (\#872488-DCPM). The funding sources had no involvements in study design; collection, analysis, and interpretation of data; or writing of the report.

\section{Footnote}

Reporting Checklist: The authors have completed the MDAR reporting checklist. Available at http://dx.doi.org/10.21037/ atm-20-4182

Data Sharing Statement: Available at http://dx.doi. org/10.21037/atm-20-4182 
Conflicts of Interest: All authors have completed the ICMJE uniform disclosure form (available at http://dx.doi. org/10.21037/atm-20-4182). NZ serves as an unpaid editorial board member of Annals of Translational Medicine from Jan 2019 to Dec 2024. ZZ receives a consulting fee from Dräger Medical. IF reports funding from the European Union's Framework Programme for Research and Innovation Horizon 2020 (WELMO, Grant No. 825572 ) and reimbursement of speaking fees, congress and travel costs by Dräger Medical. The other authors declare no conflicts of interest.

Ethical Statement: The authors are accountable for all aspects of the work in ensuring that questions related to the accuracy or integrity of any part of the work are appropriately investigated and resolved. The trial was conducted in accordance with the Declaration of Helsinki (as revised in 2013). The patients were from undergoing studies that were approved by institutional ethics review boards of the first affiliated hospital of Guangzhou Medical University, Guangzhou (2017-04) and of Tri-Service General Hospital, Taipei (2-106-05-105). Informed consent was obtained from all patients or next of kin before data were included into the study.

Open Access Statement: This is an Open Access article distributed in accordance with the Creative Commons Attribution-NonCommercial-NoDerivs 4.0 International License (CC BY-NC-ND 4.0), which permits the noncommercial replication and distribution of the article with the strict proviso that no changes or edits are made and the original work is properly cited (including links to both the formal publication through the relevant DOI and the license). See: https://creativecommons.org/licenses/by-nc-nd/4.0/.

\section{References}

1. Vyshedskiy A, Murphy R. Pendelluft in chronic obstructive lung disease measured with lung sounds. Pulm Med 2012;2012:139395.

2. Yoshida T, Torsani V, Gomes S, et al. Spontaneous effort causes occult pendelluft during mechanical ventilation. Am J Respir Crit Care Med 2013;188:1420-7.

3. Harada K, Saoyama N, Izumi K, et al. Experimental pendulum air in the flail chest. Jpn J Surg 1983;13:219-26.

4. Yoshida T, Roldan R, Beraldo MA, et al. Spontaneous Effort During Mechanical Ventilation: Maximal Injury With Less Positive End-Expiratory Pressure. Crit Care
Med 2016;44:e678-88.

5. Maloney JV, Jr., Schmutzer KJ, Raschke E. Paradoxical respiration and "pendelluft". J Thorac Cardiovasc Surg 1961;41:291-8.

6. Hedenstierna G, Bindslev L, Santesson J. Pressurevolume and airway closure relationships in each lung in anaesthetized man. Clin Physiol 1981;1:479-93.

7. Pelosi P, Cereda M, Foti G, et al. Alterations of lung and chest wall mechanics in patients with acute lung injury: Effects of positive end-expiratory pressure. Am J Respir Crit Care Med 1995;152:531-7.

8. Nakano Y, Muro S, Sakai H, et al. Computed tomographic measurements of airway dimensions and emphysema in smokers. Correlation with lung function. Am J Respir Crit Care Med 2000;162:1102-8.

9. Kauczor HU, Chen XJ, van Beek EJ, et al. Pulmonary ventilation imaged by magnetic resonance: At the doorstep of clinical application. Eur Respir J 2001;17:1008-23.

10. Tsuzaki K, Hales CA, Strieder DJ, et al. Regional lung mechanics and gas transport in lungs with inhomogeneous compliance. J Appl Physiol (1985) 1993;75:206-16.

11. Tabuchi A, Nickles HT, Kim M, et al. Acute Lung Injury Causes Asynchronous Alveolar Ventilation That Can Be Corrected by Individual Sighs. Am J Respir Crit Care Med 2016;193:396-406.

12. Frerichs I, Amato MB, van Kaam AH, et al. Chest electrical impedance tomography examination, data analysis, terminology, clinical use and recommendations: consensus statement of the TRanslational EIT developmeNt stuDy group. Thorax 2017;72:83-93.

13. Rossi FS, Costa ELV, Iope DDM, et al. Pendelluft Detection Using Electrical Impedance Tomography in an Infant. Keep Those Images in Mind. Am J Respir Crit Care Med 2019;200:1427-9.

14. Santini A, Mauri T, Dalla Corte F, et al. Effects of inspiratory flow on lung stress, pendelluft, and ventilation heterogeneity in ARDS: A physiological study. Crit Care 2019;23:369.

15. Morais CCA, Koyama Y, Yoshida T, et al. High Positive End-Expiratory Pressure Renders Spontaneous Effort Noninjurious. Am J Respir Crit Care Med 2018;197:1285-96.

16. Delgado DA, Lambert BS, Boutris N, et al. Validation of Digital Visual Analog Scale Pain Scoring With a Traditional Paper-based Visual Analog Scale in Adults. J Am Acad Orthop Surg Glob Res Rev 2018;2:e088.

17. ARDS Definition Task Force, Ranieri VM, Rubenfeld GD, et al. Acute respiratory distress syndrome: The Berlin 
Definition. Jama 2012;307:2526-33.

18. Vogelmeier CF, Criner GJ, Martinez FJ, et al. Global Strategy for the Diagnosis, Management, and Prevention of Chronic Obstructive Lung Disease 2017 Report. GOLD Executive Summary. Am J Respir Crit Care Med 2017;195:557-82.

19. Reddel HK, Levy ML. The GINA asthma strategy report: what's new for primary care? NPJ Prim Care Respir Med 2015;25:15050.

20. Zhang N, Jiang H, Zhang C, et al. The influence of electrical impedance tomography belt on lung function determined by spirometry in sitting position. Physiol Meas 2020;41:044002.

21. Yoshida T, Uchiyama A, Matsuura N, et al. Spontaneous breathing during lung-protective ventilation in an experimental acute lung injury model: high transpulmonary pressure associated with strong spontaneous breathing effort may worsen lung injury. Crit Care Med 2012;40:1578-85.

22. Pulletz S, Adler A, Kott M, et al. Regional lung opening and closing pressures in patients with acute lung injury. J Crit Care 2012;27:323.e11-8.

Cite this article as: Sang L, Zhao Z, Yun PJ, Frerichs I, Möller K, Fu F, Liu X, Zhong N, Li Y. Qualitative and quantitative assessment of pendelluft: a simple method based on electrical impedance tomography. Ann Transl Med 2020;8(19):1216. doi: 10.21037/atm-20-4182
23. Karagiannidis C, Waldmann AD, Roka PL, et al. Regional expiratory time constants in severe respiratory failure estimated by electrical impedance tomography: A feasibility study. Crit Care 2018;22:221.

24. Frerichs I, Zhao Z, Becher T, et al. Regional lung function determined by electrical impedance tomography during bronchodilator reversibility testing in patients with asthma. Physiol Meas 2016;37:698-712.

25. Vogt B, Zhao Z, Zabel P, et al. Regional lung response to bronchodilator reversibility testing determined by electrical impedance tomography in chronic obstructive pulmonary disease. Am J Physiol Lung Cell Mol Physiol 2016;311:L8-19.

26. Zhao Z, Chang MY, Frerichs I, et al. Regional air trapping in acute exacerbation of obstructive lung diseases measured with electrical impedance tomography: A feasibility study. Minerva Anestesiol 2020;86:172-80.

27. Zhao Z, Peng SY, Chang MY, et al. Spontaneous breathing trials after prolonged mechanical ventilation monitored by electrical impedance tomography: An observational study. Acta Anaesthesiol Scand 2017;61:1166-75. 